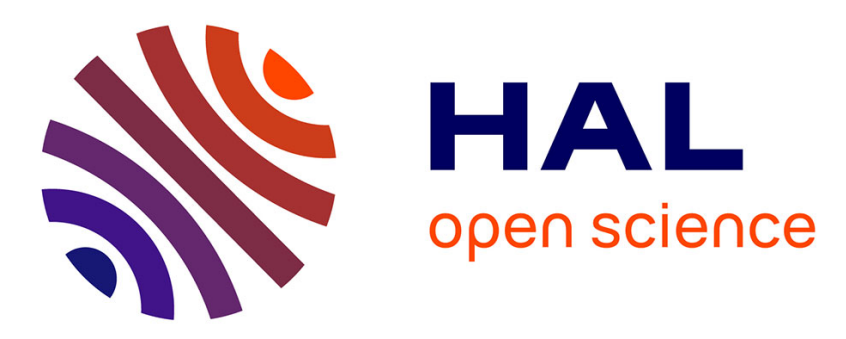

\title{
Robust autonomous robot tracking using interval analysis
}

Michel Kieffer, Luc Jaulin, Eric Walter, Dominique Meizel

\section{To cite this version:}

Michel Kieffer, Luc Jaulin, Eric Walter, Dominique Meizel. Robust autonomous robot tracking using interval analysis. IFAC-SYSID 2000, System Identification Congress 2000, Jun 2000, Santa-Barbara

(California), United States. pp.x-x. hal-00844976

\section{HAL Id: hal-00844976 \\ https://hal.science/hal-00844976}

Submitted on 16 Jul 2013

HAL is a multi-disciplinary open access archive for the deposit and dissemination of scientific research documents, whether they are published or not. The documents may come from teaching and research institutions in France or abroad, or from public or private research centers.
L'archive ouverte pluridisciplinaire HAL, est destinée au dépôt et à la diffusion de documents scientifiques de niveau recherche, publiés ou non, émanant des établissements d'enseignement et de recherche français ou étrangers, des laboratoires publics ou privés. 


\title{
ROBUST AUTONOMOUS ROBOT TRACKING USING INTERVAL ANALYSIS

\author{
Michel KIEFFER *, Luc JAULIN *,**, Éric WALTER* \\ and Dominique MEIZEL ${ }^{* * *}$
}

\author{
${ }^{*}$ Laboratoire des Signaux et Systèmes, \\ CNRS - Supélec - Université Paris-Sud \\ Plateau de Moulon, 91192 Gif-sur-Yvette, France \\ \{jaulin,kieffer,walter\}@lss.supelec.fr \\ ** on leave from Laboratoire d'Ingénierie des Systèmes \\ Automatisés, Université d'Angers, France \\ *** HEUDIASYC, CNRS, Université de Technologie de \\ Compiègne, B.P. 20529, 60205 Compiègne, France \\ meizel@hds.univ-compiegne.fr
}

\begin{abstract}
This paper deals with robust and autonomous robot tracking using distance measurements provided by a belt of on-board ultrasonic sensors. The measurements errors are assumed to be bounded. The method presented uses a set-valued nonlinear state estimator. As a Kalman filter, it alternates prediction based on past data and correction to take new measurements into account. Special attention is paid to the treatment of outliers due, e.g., to a partially outdated map or to faulty sensors. Copyright ${ }^{\circledR} 2000$ IFAC
\end{abstract}

Keywords: intervals, localization, parameter estimation, robotics, state estimation, tracking

\section{INTRODUCTION}

This paper deals with robust and autonomous robot tracking using distance measurements provided by a belt of on-board ultrasonic sensors. The robot represented on Figure 1 has to localize itself in a 2D environment, a map of which is available.

The measurements errors are assumed to be bounded. The method presented uses a set-valued nonlinear state estimator (Kieffer et al., 1998). As a Kalman filter, it alternates prediction based on past data and correction to take new measurements into account. The correction step involves a robust localization technique developed for static robot localization and presented in (Kieffer et al., 1999), (Kieffer et al., 2000). These two contributions are briefly recalled and special attention is paid to the treatment of outliers due, e.g., to a partially outdated map or to faulty sensors.

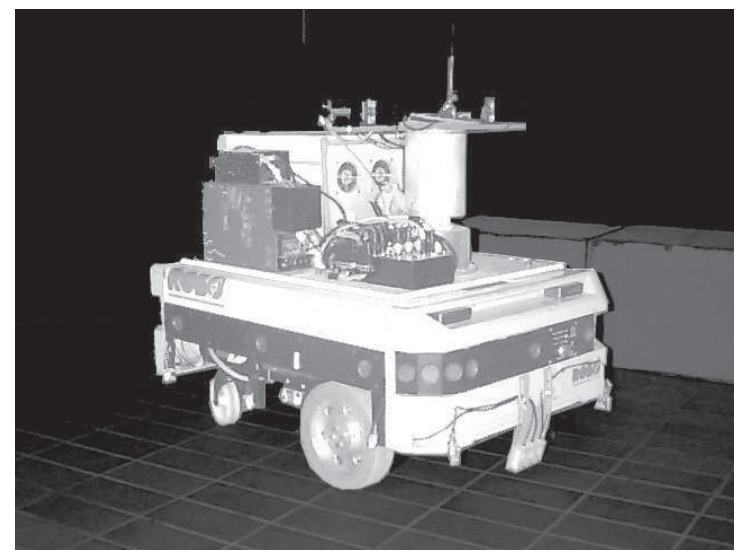

Fig. 1. The Robuter mobile robot 


\section{STATIC LOCALIZATION}

The aim of static localization is the evaluation of the configuration vector $\mathbf{x}$ of a motionless robot. This vector consists of the position $\left(x_{\mathrm{c}}, y_{\mathrm{c}}\right)$ and orientation $\theta$ of the robot in a given world frame attached to its environment.

The localization procedure will be used in the correction step of the recursive state estimation algorithm.

\subsection{Information}

The map of the environment consists of a collection of segments representing landmarks (walls, pillars, etc.). An example of such a map is represented on Figure 2.

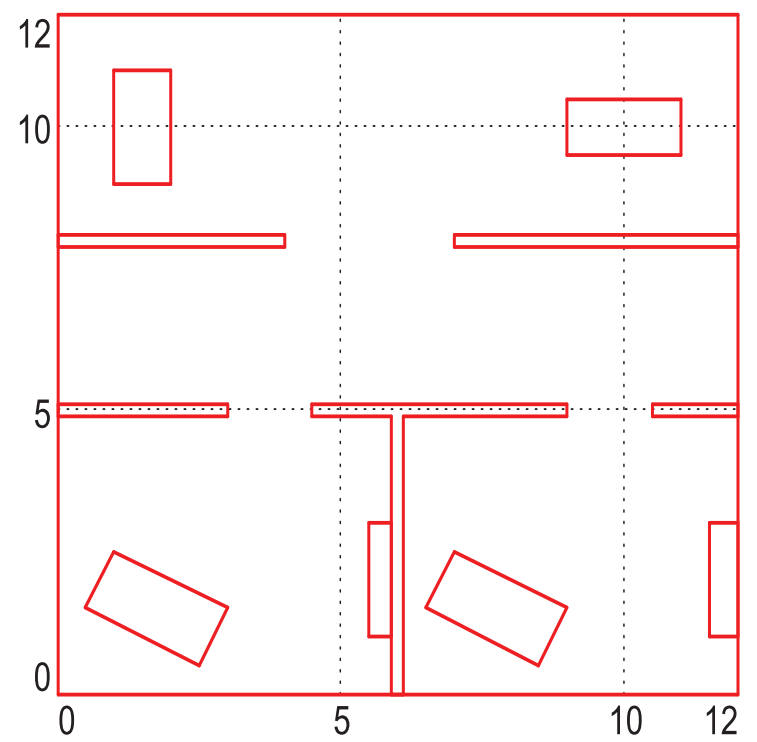

Fig. 2. Map of the environment of the robot; the distances are indicated in meters

Each ultrasonic sensor $i$ emits a wave in a given direction. This wave is assumed to propagate in a cone with half-aperture $\gamma$. The distance $d_{i}$ to the closest landmark situated in the emission cone and such that the incidence angle is lower than $\beta+\gamma$ is evaluated. The diffuse reflexion angle $\beta$ is a characteristic of the surface of the material composing the obstacle. Figure 3 illustrates the two possible situations. In (a) the incidence angle of the ultrasonic wave is lower than $\beta+\gamma$, so the reflected part of the ultrasonic wave is caught by the sensor. In (b) the incidence angle is greater than $\beta+\gamma$, and no reflected wave will be received directly by the sensor. The sensor may still be hit after multiple reflexions, but the measurement reported will then no longer correspond to the distance from the sensor to the closest landmark in the emission cone.

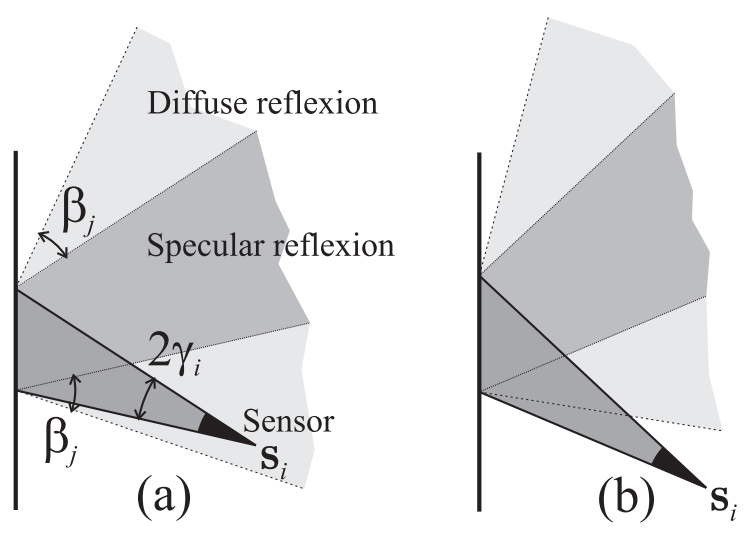

Fig. 3. (a) Valid distance measurement;

(b) invalid distance measurement

To take measurement inaccuracy into account, an interval $\left[d_{i}\right]=\left[(1-\alpha) d_{i},(1+\alpha) d_{i}\right]$ is considered. The values of $\gamma, \alpha$ and $\beta$ are obtained by experimentation on the robot and on the material composing the environment. An emission diagram such as that represented on Figure 4 summarizes the uncertain distance measurements provided by the sensors. For each measurement, a landmark should be at least partially located between the two arcs of circles representing the uncertainty on each measurement.

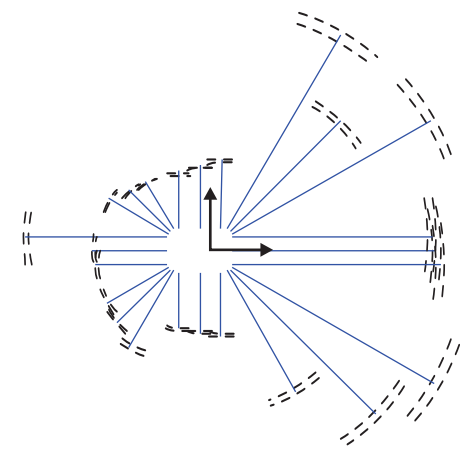

Fig. 4. An emission diagram

Remark 2.1. For the sake of simplicity, the values of $\alpha$ and $\gamma$ have been assumed here to be the same for all sensors, but the method could deal easily with differentiated sensors. Similarly, the angle $\beta$ may depend on the material hit by the ultrasonic wave. Information on the value of $\beta$ for each segment could easily be incorporated in the map to lead to a more accurate description.

\subsection{Set inversion}

The configuration vector $\mathbf{x}$ is assumed to belong to some search box (or interval vector) $\left[\mathbf{x}_{0}\right]$. We are looking for the set

$$
\mathcal{S}=\left\{\mathbf{x} \in\left[\mathbf{x}_{0}\right] \mid t(\mathbf{x}) \text { is true }\right\},
$$


where $t(\mathbf{x})$ is a test stating that $\mathbf{x}$ is compatible with the map and the uncertain measurements provided by the sensors. The test $t(\mathbf{x})$ consists of a conjunction of elementary tests $t_{i}(\mathbf{x})$, each associated with a sensor.

$$
t(\mathbf{x})=t_{1}(\mathbf{x}) \wedge \ldots \wedge t_{n_{\mathrm{s}}}(\mathbf{x}),
$$

where $\wedge$ represents the logical and and $n_{\mathrm{s}}$ is the number of sensors. For the $i^{\text {th }}$ sensor, $t_{i}(\mathbf{x})$ is built by simulating the telemetric process. The distance that the $i^{\text {th }}$ sensor would report if the robot were in the configuration $\mathbf{x}$ is evaluated. If the result belongs to $\left[d_{i}\right]$, which is assumed to contain the actual distance, the configuration is compatible with the $i^{\text {th }}$ measurement given the map.

The test $t(\mathbf{x})$ holds true if $\mathbf{x}$ is compatible with all the measurements given the map. For more details on how $t(\mathbf{x})$ is evaluated, see (Kieffer, 1999) or (Kieffer et al., 2000). In this paper, the test has been improved to take the effect of the reflexion angle $\beta$ into account.

Characterizing $\mathcal{S}$ using (1) is a set inversion problem, which is solved in an approximated but guaranteed way using SiviA (for Set Inversion Via Interval Analysis) (Jaulin and Walter, 1993). The set $\mathcal{S}$ is enclosed in a union of nonoverlapping boxes (or subpaving) $\widehat{\mathcal{S}}$. This outer approximation can be made as precise as desired. It is guaranteed, in the sense that all the configurations compatible with the available information belong to it.

\subsection{Robustness to outliers}

Ultrasonic sensors are cheap but unreliable. Erroneous measurements, or outliers, are frequent, due, e.g., to multiple reflections, sensor failures, outdated maps, etc. In the presence of such outliers, the set $\mathcal{S}$, as defined by (1), may turn out to be empty. Using the q-relaxed and Boolean operator, denoted by $\bigoplus_{q}$, it is possible to tolerate up to $q$ outliers by characterizing the set

$$
\mathcal{S}_{q}=\left\{\mathbf{x} \in\left[\mathbf{x}_{0}\right] \mid t_{\text {outliers }}(\mathbf{x}, q) \text { is true }\right\},
$$

where

$$
\begin{aligned}
t_{\text {outliers }}(\mathbf{x}, q) & =\bigoplus_{q}\left(t_{1}(\mathbf{x}), \ldots, t_{n_{\mathrm{s}}}(\mathbf{x})\right) \\
& =\bigoplus_{i=1}^{n_{\mathrm{s}}} q\left(t_{i}(\mathbf{x})\right)
\end{aligned}
$$

holds true if at least $n_{\mathrm{s}}-q$ of the tests $t_{i}(\mathbf{x})$ do.

A possible policy is to start with $q=0$, which corresponds to using (1), and to increase $q$ by one whenever the set of possible configurations is found empty. This corresponds to a guaranteed implementation of the Outlier Minimal Number Estimator (OMNE) ((Lahanier et al., 1987), (Pronzato and Walter, 1996), and (Walter and Piet-Lahanier, 1988)). For more details, see (Jaulin et al., 1996), (Kieffer et al., 1999) and (Kieffer et al., 2000).

\subsection{Example}

The robot is placed in an environment, the map of which is represented on Figure 2. The (unknown) configuration of the robot is $\left(x_{c}, y_{c}, \theta\right)=$ $(6 \mathrm{~m}, 3.5 \mathrm{~m}, 6.10 \mathrm{rad})$. The distance measurements provided by the on-board sensors corresponds to the emission diagram of Figure 4. Simulated data were obtained taking $\alpha=0.02, \gamma=0.1978 \mathrm{rad}$ and $\beta=0.5 \mathrm{rad}$. Three outliers have been introduced to simulate sensors failures.

The search box $\left[\mathbf{x}_{0}\right]$ is taken equal to $[0 \mathrm{~m}, 12 \mathrm{~m}] \times$ $[0 \mathrm{~m}, 12 \mathrm{~m}] \times[0 \mathrm{rad}, 2 \pi \mathrm{rad}]$. The static localiza-

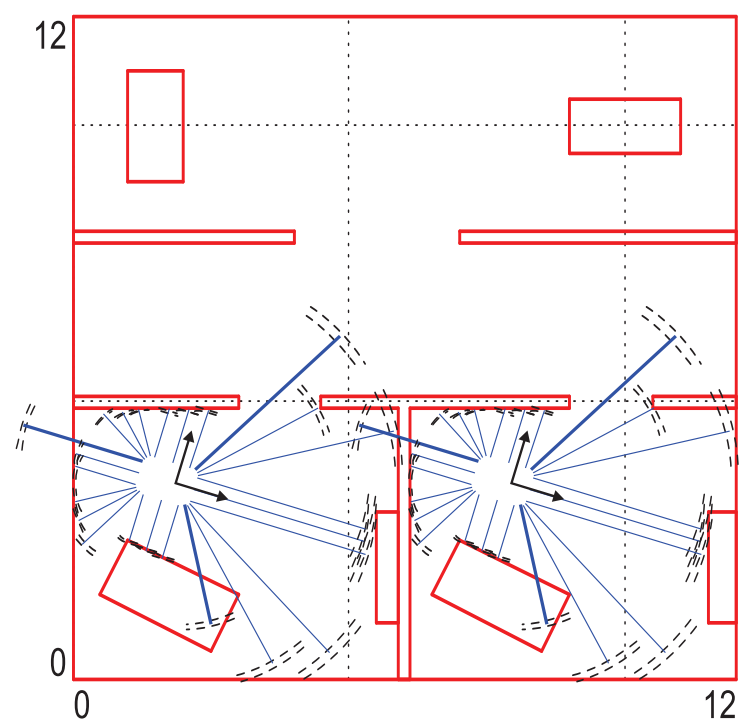

Fig. 5. Two of the possible configurations obtained by the static localization procedure

tion procedure returns no solution till at least three outliers are tolerated. When $q=3$, the solution set $\widehat{\mathcal{S}}$ consists of two disconnected subsets, which are guaranteed to contain the actual configuration provided there are no more than three outliers. Figure 5 displays two configurations belonging to the solution subpaving $\widehat{\mathcal{S}}$ represented on Figure 6, for $q=3$. The measurements corresponding to outliers have been represented in bold.

When $q$ is increased, the volume of the solution set may increase. Table 1 summarizes the computing times for the localization procedure, for $q=0$ up to 4 , on a Pentium-II $450 \mathrm{MHz}$ personnal computer. 


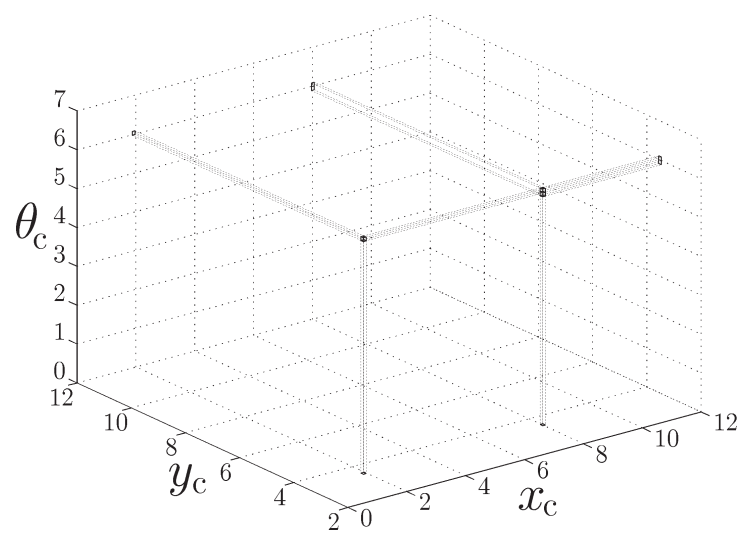

Fig. 6. Solution set of the static localization procedure, with $q=3$, and its projections

\begin{tabular}{c|c|c|c}
$q$ & Volume of $\widehat{\mathcal{S}}$ & Comput. time & Cumul. c. t. \\
\hline \hline 0 & 0 & 97 sec. & 97 sec. \\
1 & 0 & 144 sec. & 241 sec. \\
2 & 0 & 189 sec. & 430 sec. \\
3 & 0.004 & 275 sec. & 705 sec. \\
4 & 0.011 & 346 sec. & 1051 sec.
\end{tabular}

Table 1: Result of static localization

\section{TRACKING}

The robot may now be moving. Our aim is then to estimate its configuration (or state) as a function of time. Only the initial static localization results and measurements obtained during motion may be taken into account.

The state $\mathbf{x}$ of the robot and the measurements $\mathbf{y}$ satisfy a nonlinear model

$$
\left\{\begin{array}{l}
\mathbf{x}_{l+1}=\mathbf{f}_{l}\left(\mathbf{x}_{l}, \mathbf{u}_{l}, \mathbf{v}_{l}\right), \quad l=0,1, \ldots, \\
\mathbf{y}_{l}=\mathbf{h}_{l}\left(\mathbf{x}_{l}\right)+\mathbf{w}_{l},
\end{array}\right.
$$

where $\mathbf{u}_{l} \in \mathbb{R}^{m}, \mathbf{x}_{l} \in \mathbb{R}^{n}$ and $\mathbf{y}_{l} \in \mathbb{R}^{p}$ are respectively the input, state, and measurement vectors. This model is obtained by exact discretization of the kinematic equations of the robot; the state perturbation $\mathbf{v}_{l}$ takes, e.g., unmodelled frictions into account. The robot is steered by the independently adjustable speeds of two of its wheels, which are characterized by the input vector $\mathbf{u}_{l}$.

The initial state $\mathbf{x}_{0}$ is assumed to be included in some prior compact set $\mathcal{X}_{0} \subset \mathbb{R}^{n} .\left\{\mathbf{v}_{l}\right\}$ and $\left\{\mathbf{w}_{l}\right\}$ are unknown state perturbation and measurement noise sequences, assumed to belong respectively to the known intervals sequences $\left\{[\mathbf{v}]_{l}\right\}$ and $\left\{[\mathbf{w}]_{l}\right\}$. $\mathbf{f}_{l}$ and $\mathbf{h}_{l}$ are known functions (or finite algorithms).

\subsection{Recursive nonlinear state estimator}

An idealized version of the state estimator will first be presented. Consider a set $\mathcal{X}_{l}$ containing all possible values of the state vector $\mathbf{x}_{l}$ at time $l$. At time $l+1$, a set $\mathcal{X}_{l+}$ containing all the values of the state vector that are accessible from an $\mathbf{x}_{l}$ taken in $\mathcal{X}_{l}$ is evaluated using the state equation in (4). This yields set (1) in Figure 7 . Note that $\mathcal{X}_{l}$ and $\mathcal{X}_{l+}$ may both consist of disconnected subsets.

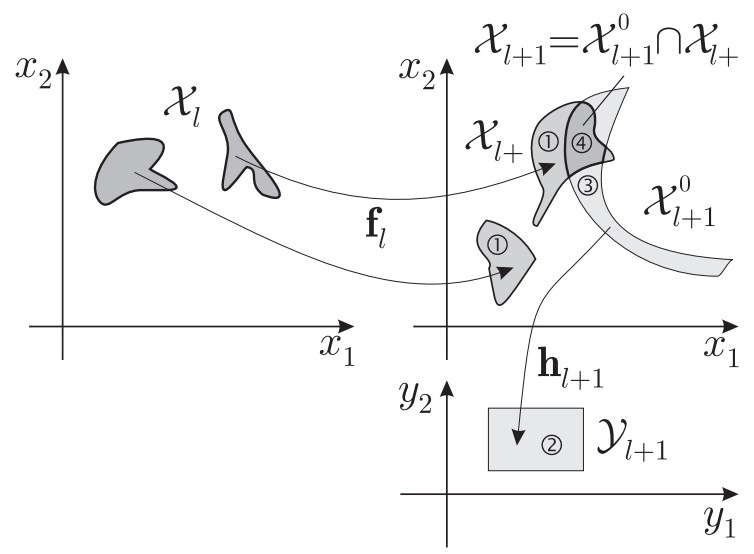

Fig. 7. Idealized state estimation algorithm

Suppose that at time $l+1$, a measurement vector $\mathbf{y}_{l+1}$ becomes available. Taking the bounds on measurement errors into account, the output may belong to a set $\mathcal{Y}_{l+1}$ (see Figure 7 - set (2)). In the observation equation in (4), the function $\mathbf{h}_{l+1}$ describes how the measurement are obtained (here, the function used to build the tests $t_{i}(\mathbf{x})$ will be used). The state should thus belong to the set $\mathcal{X}_{l+1}^{o}=\mathbf{h}_{l+1}^{-1}\left(\mathcal{Y}_{l+1}\right)$ (see Figure 7 - set (3)).

The set $\mathcal{X}_{l+1}$ of all the state vectors $\mathbf{x}_{l+1}$ compatible with the information available at time $l+1$ is then the intersection of $\mathcal{X}_{l+}$ and $\mathcal{X}_{l+1}^{o}$ (see Figure 7 - set (4)).

Figure 7 thus illustrates the two basic steps of the recursive nonlinear state estimation algorithm. The prediction step evaluates how the set containing the state evolves and the correction step takes newly available information into account to discard parts of the predicted set.

In general, this idealized algorithm is not implementable. It can however be used to derive an approximated but guaranteed state estimator based on interval analysis (see (Kieffer, 1999) and (Kieffer et al., 1998)).

\subsection{Application to robust robot tracking}

The resulting nonlinear state estimator is applied to the tracking of the robot from measurements that may contain outliers.

The prediction step uses an exact discretization of the kinematic equations of the robot. Although no state perturbation has been considered in the example of Section 3.3, it could easily be incorporated in the motion equations. 
The correction step is implemented using the static localization procedure presented in Section 2. Only the configuration subspace corresponding to the set obtained during the previous prediction step is explored, which leads to a spectacular speeding up of the localization procedure.

Concerning the number of outliers that may be tolerated during tracking, various policies may be considered. At each step, one may begin with $q=0$ and increase $q$ as long as the corrected set remains empty. Another strategy would fix $q=q_{\text {up }}$, some bound of the tolerated number of outliers. This second technique is less timeconsuming, but also less efficient in eliminating subsets that cannot contain the actual state.

\subsection{Example}

The environment, initial configuration and first measurements remain the same as in the static localization example. The tracking algorithm is initialized with the results obtained by the localization procedure. The robot actually moves from the room located on the right of the bottom of Figure 2 to that on top. During motion, new measurements are taken every second to correct the predicted set containing the state of the robot. The second strategy is applied and the maximum number of tolerated outliers $q=q_{\text {up }}$ is taken equal to eight.

The evolution of the projection of the solution set onto the $(x, y)$ plane is presented on the top of Figure 8. On the bottom of the same figure, the robot has been represented in configurations belonging to the solution set. Until step 7, this set consists of two disconnected parts, both of which may contain the actual state of the robot. After this step, there are more than eight outliers for each configuration belonging to the left subset, which is then eliminated.

The entire procedure takes about 20 sec. on a Pentium-II personnal computer at $450 \mathrm{MHz}$, which allows real time implementation.

\section{CONCLUSIONS}

The procedure advocated here does not suffer some of the traditional drawbacks of previous localization methods. It delivers a guaranteed solution, unlike the methods based on extended Kalman filtering (see (Crowley, 1989), (Leonard and Durrant-Whyte, 1991) and (Leonard and Durrant-Whyte, 1992)) or bounded-error techniques requiring a linearization, such as described in (Bertsekas and Rhodes, 1971), (Durieu et al., 1996), (Maksarov and Norton, 1996) and (Schweppe, 1973). It does not need a separate matching algorithm to recognize the environment prior to localization, contrary to (Drumheller, 1987) and (Grimson and Lozano-Pérez, 1987). It directly manages multiple hypotheses contrary to (Halbwachs and Meizel, 1997). It does not request any separate initialization procedure, contrary to (Leonard and Durrant-Whyte, 1991) and (Neira et al., 1996).

The main drawback of this procedure is that its complexity is exponential in the dimension of $\mathbf{x}$, which poses no problem in this case of robot tracking.

A demonstration of the robot localization and tracking can be found at

http://www.supelec.fr/lss/fr/personnels/kieffer/robotloc.htm

Acknowledgment: The authors express their gratitude to INTAS for partial support (Grant INTAS-RFBR-97-10782).

\section{REFERENCES}

Bertsekas, D. P. and I. B. Rhodes (1971). Recursive state estimation for a set-membership description of uncertainty. IEEE Trans. on Automatic Control 16, 117-128.

Crowley, J. (1989). World modeling and position estimation for a mobile robot using ultrasonic ranging. In: Proc. IEEE Int. Conf. on Robotics and Automation. Scottsdale, Arizona. pp. $674-680$.

Drumheller, M. (1987). Mobile robot localization using sonar. IEEE Trans. on Pattern Analysis and Machine Intelligence $\mathbf{9}(2), 325-332$.

Durieu, C., B. Polyak and E. Walter (1996). Trace versus determinant in ellipsoidal outer bounding with application to state estimation. In: Proc. 13th IFAC World Congress. Vol. I. San Francisco. pp. 43-48.

Grimson, W. E. and T. Lozano-Pérez (1987). Localizing overlapping parts by searching the interpretation tree. IEEE Trans. on Pattern Analysis and Machine Intelligence 9(4), 469482.

Halbwachs, E. and D. Meizel (1997). Multiple hypothesis management for mobile vehicule localization. In: $C D$ Rom of the European Control Conference. Louvain.

Jaulin, L. and E. Walter (1993). Set inversion via interval analysis for nonlinear bounded-error estimation. Automatica 29(4), 1053-1064.

Jaulin, L., E. Walter and O. Didrit (1996). Guaranteed robust nonlinear parameter bounding. In: Proc. CESA'96 IMACS Multiconference (Symposium on Modelling, Analysis and Simulation). Lille. pp. 1156-1161. 


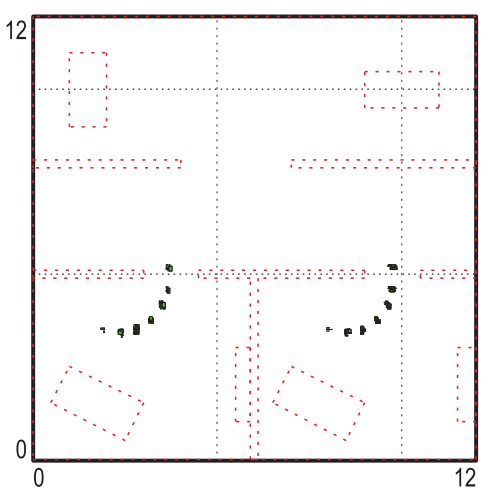

$t=6 \mathrm{sec}$.

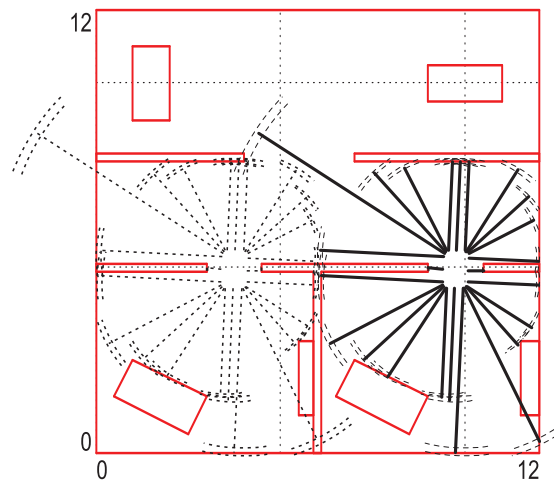

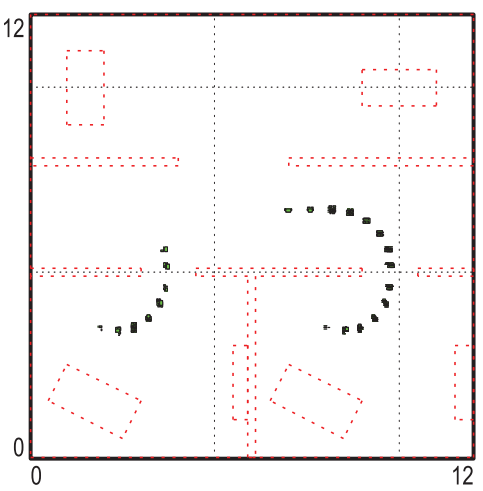

$t=14 \mathrm{sec}$.

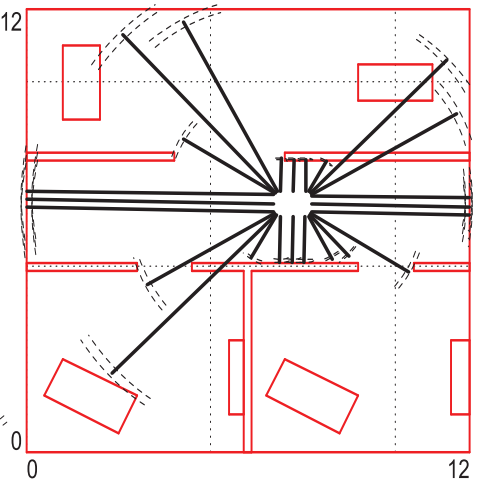

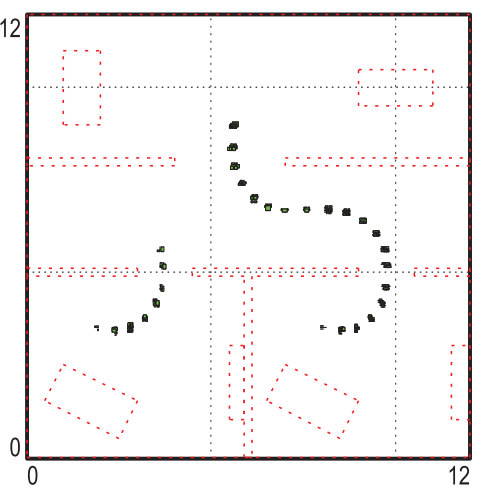

$t=19 \mathrm{sec}$.

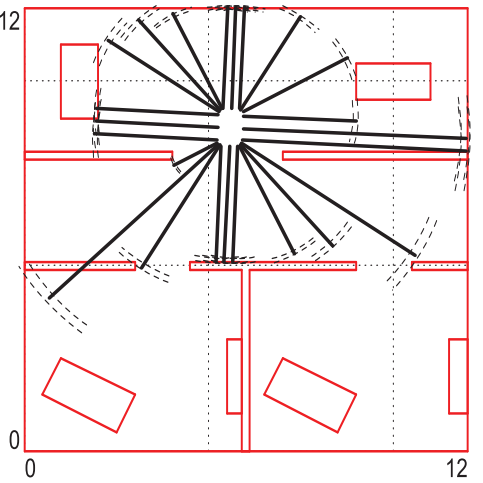

Fig. 8. Evolution of the projection on the $(x, y)$ - plane of the solution set, and possible configuration; measurements are collected every second

Kieffer, M. (1999). Estimation ensembliste par analyse par intervalles, application à la localisation d'un véhicule. PhD dissertation. Université Paris-Sud. Orsay.

Kieffer, M., L. Jaulin and E. Walter (1998). Guaranteed recursive nonlinear state estimation using interval analysis. In: Proc. 37th IEEE Conference on Decision and Control. Tampa. pp. 3966-3971.

Kieffer, M., L. Jaulin, E. Walter and D. Meizel (1999). Nonlinear identification based on unreliable priors and data, with application to robot localization. In: Robustness in Identification and Control (A. Garulli, A. Tesi and A. Vicino, Eds.). Springer. London. pp. 190203, LNCIS 245.

Kieffer, M., L. Jaulin, E. Walter and D. Meizel (2000). Robust autonomous robot localization using interval analysis. To appear in $R e-$ liable Computing 6(3).

Lahanier, H., E. Walter and R. Gomeni (1987). OMNE: a new robust membership-set estimator for the parameters of nonlinear models. J. of Pharmacokinetics and Biopharmaceutics 15, 203-219.

Leonard, J. J. and H. F. Durrant-Whyte (1991). Mobile robot localization by tracking geometric beacons. IEEE Trans. on Robotics and Automation 7(3), 376-382.

Leonard, J. J. and H. F. Durrant-Whyte (1992). Directed Sonar Sensing for Mobile Robot
Navigation. Kluwer Academic Publishers. Boston.

Maksarov, D. and J. P. Norton (1996). State bounding with ellipsoidal set description of the uncertainty. Int. J. of Control 65(5), 847866.

Neira, J., J. Horn, J. D. Tardoz and G. Schmidt (1996). Multisensor mobile robot localization. In: Proc. IEEE International Conference on Robotics and Automation. Mineapolis. pp. 673-679.

Pronzato, L. and E. Walter (1996). Robustness to outliers of bounded-error estimators and consequences on experiment design. In: Bounding Approaches to System Identification (M. Milanese, J. Norton, H. PietLahanier and E. Walter, Eds.). Plenum Press. New York. pp. 199-212.

Schweppe, F. C. (1973). Uncertain Dynamic Systems. Prentice-Hall. Englewood Cliffs, NJ.

Walter, E. and H. Piet-Lahanier (1988). Estimation of the parameter uncertainty resulting from bounded-error data. Mathematical Biosciences 92, 55-74. 